\title{
Survival rates similar with full cast crowns and direct composite restorations
}

\author{
In patients with endodontically treated premolars are full cast crowns more \\ effective than direct composite restorations?
}

\begin{abstract}
Mannocci F, Bertelli E, Sherriff M, Watson TF, Pitt Ford TR. Three-year clinical comparison of survival of endodontically treated teeth restored with either full cast coverage or with direct composite restoration. J Prosthet Dent 2002; 88:297-301
\end{abstract}

Design A 3-year pseudo-randomised controlled trial was conducted. Intervention Subjects were randomly assigned by coin toss to either endodontic treatment and restoration with adhesive techniques and composite, or endodontic treatment and restoration with adhesive techniques and composite with final restoration with full-coverage metal-ceramic crown.

Outcome measure Restoration survival or failure was recorded. Causes of failure were categorised as root fracture, post fracture, post decementation, clinical and/or radiographic evidence of marginal gap between tooth and restoration, and clinical and/or radiographic evidence of secondary caries contiguous with restoration margins.

Results At 3 years there was no difference in the failure frequencies between the two groups.

Conclusions Clinical success rates of endodontically treated premolars restored with fibre posts and direct composite restorations after 3 years of service were equivalent to a similar treatment of full coverage with metal-ceramic crowns.

\section{Commentary}

The decreased strength seen in endodontically treated teeth is primarily because of the loss of coronal tooth structure. ${ }^{1}$ Thus, coronal restoration in these teeth is an essential step in the evaluation of long-term success. Tooth structure loss can range from minimal access preparation in intact teeth to extensive damage that endangers the longevity of the tooth itself. ${ }^{2}$ Little documentation exists regarding the description and outcome of endodontically treated teeth restored with posts that have been covered with metal-ceramic crowns or left uncovered. This finding is surprising given the clinical prevalence of this procedure.

In this study, Mannocci et al. performed a randomised controlled clinical study to evaluate the clinical success rate of endodontically treated premolars. The question the authors addressed was whether teeth with limited loss of tooth structure that had endodontic treatment and core buildup with adhesive techniques, fibre posts, and composites, would demonstrate equivalent failure rates and failure modes to teeth restored in a similar manner with full crown coverage.

The investigators enrolled 117 subjects for their study. Although, the setting (university, hospital, private practice) was not specified in the paper, the description of the population in terms of inclusion and exclusion criteria and patients' characteristics were clearly described. The patients were assigned in a randomised manner but the allocation method was not concealed.
The description of the intervention was described clearly and so as to be reproducible. One operator carried out the complete procedure. The treatment performed was the same in all the patients in the group. Co-interventions (concomitant treatments) were not mentioned. The outcomes were measured before restoration, immediately after restoration and at recall after 1,2 and 3 years.

Success or failure was evaluated using clinical, radiographic and photographic examination. Two examiners, neither the operator, carried out the assessments. The results showed no significant differences between the groups. There were 30 withdrawals (a $26 \%$ loss to follow-up). The causes of the withdrawals and dropouts were not explained. The majority of these withdrawals (27 individuals) were from the same group. This was an important point that should have been discussed - this loss to follow-up virtually negates the results. On other hand, using a worst-case scenario analysis (the withdrawals are considered 'failures'), the results show 30\% failures after the second year for group 1 and $11 \%$ in group 2 . After the third year, $20 \%$ failures were found in group 1 and $5.2 \%$ in group 2.

The study highlights the importance of degree of tooth structure loss when planning the final restoration of endodontically treated teeth. The results here may help with decision-making in clinical practice regarding differences in time and cost of the treatments. The research was relatively well designed but there were some deficiencies. The study should have been designed to include matched pairs of teeth in the same patients; the follow up-time may have been insufficient; and no sample size or power calculation means that there may not have been enough subjects/teeth to find a difference if there really was one. The authors did, however, identify some of these limitations in their discussion.

\section{Practice point}

- Endodontically treated teeth with minimal loss of coronal structure may be restored with adhesion techniques.

\section{Bettina Basrani ${ }^{1}$, Debora Matthews ${ }^{2}$}

${ }^{1}$ Endodontic Division, Dalhousie University, Halifax, Nova Scotia, Canada

${ }^{2}$ Periodontic Division, Dalhousie University, Halifax, Nova Scotia, Canada

1. Reeh ES, Messer HH, Douglas WH. Reduction in tooth stiffness as a result of endodontic and restorative procedures. J Endod 1989; 15:512-516.

2. Gutmann JL. The dentin-root complex: anatomic and biologic considerations in restoring endodontically treated teeth. J Prosthet Dent 1992; 67:458-467.

Evidence-Based Dentistry (2004) 5, 45.

doi:10.1038/sj.ebd. 6400253 